\title{
Initial Flight Simulation Testing of a Gesture-based Interface for Flight Control
}

\author{
M. A. Rodríguez Díaz, E. Villani, D. Arjoni, P. Krus* \\ Aeronautics Institute of Technology, São José dos Campos, Brazil. \\ E-mail: manuelrd@ita.br, evillani@ita.br, diegoarjoni@gmail.com, petter.krus@liu.se \\ *Linköping University, Linköping, Sweden.
}

\begin{abstract}
As our knowledge in aeronautics has improved, so have aircraft. Modern flight control systems can use electronic systems to greatly enhance the controllability, efficiency and safety of even aerodynamically unstable aircraft, however the man-machine interface method has remained the same since the early days; sticks and pedals. This paper depicts the very first simulated test flights on a prototype flight control interface for manned aircraft under development, this interface uses the pilot's eye focus direction (known as "gaze") and hand movements to control the aircraft in flight by just looking into a desired direction, or by having the aircraft to "imitate" her or his hand like one would do to illustrate an aircraft flight maneuver: an open hand with thumb and pinky finger extended like "wings" to imitate an aircraft motion in flight, except that this time is the other way around, with the aircraft following the hand rolls and climbs as actual commands. Although Gaze-guided and Hand gesture-guided flight control has been proven feasible using this system, only the initial operative experience is covered, with a general overview on the systems functionality and of data collected, this exploratory research does not intends to result in a immediately-applicable system, rather as proof of concept of the interface method of the proposed system and as foundation for further development.
\end{abstract}

Keywords: Flight Controls, Eye Tracking, Body Tracking, Control Systems.

\section{Introduction}

Along more than one century of research and development in Aeronautics, many innovations have been introduced. Examples are the use of composite materials and the introduction of fly-by-wire control systems [1].

Fly-by-wire systems have made possible the use of new aerodynamic design configurations, with different shapes, purposes and capabilities [2]. Revolutionary as these aircrafts might be, a proficient pilot from an early biplane would have no issue controlling them. This is possible because he would still use a thrust lever for controlling power, a yoke or side stick for controlling pitch and roll, and a pair of pedals for controlling yaw. The main aircrafts control interfaces have not changed in concept since the first heavier-than-air airplanes.

Despite advances in technology and the introduction of flyby-wire systems, aircraft control interfaces have remained largely unchanged. Current interfaces are limited to 3 degrees of freedom for each control as a maximum. Most commonly, they use only one or two degrees of freedom, be it a stick or a pedal.

A vehicle like a rotorcraft can move in 6 degrees of freedom in normal conditions. New control interfaces using current technologies could provide more controllability of the craft than existing interfaces.

They could also reduce the training required to operate a flying vehicle. This last point can be of particular interest for the personal flying vehicles current under development by major aerospace corporations [3] [4] [5]. While their conception calls for them to be self-piloted, the capability for a human to intervene in case of a navigation failure could be important for safety reasons.

Considering the above motivation, this paper presents the development of new control interfaces that do not require yokes, sticks or pedals for inputs on the aircraft roll, pitch, yaw and thrust controls. Instead, it explores the use of body motion and visual focus direction.

The paper is organized as follows. Section 2 presents the architecture of the proposed system. Section 3 presents the flight control modes, Section 4 describes the experimental simulated flights that were performed to collect data, while Section 5 Presents the results from these experiments. Finally, Section 6 presents the conclusions. 


\section{The System Architecture}

The proposal presented in this paper uses two types of signal inputs for controlling the aircraft: body motion and visual focus direction.

In the first case, the movements of the pilot's hand are captured using components from the HTC Vive virtual reality kit. This equipment relies on a system of rotating laser beam base stations ("lighthouses", as these are known) to perform spatial tracking. It also uses accelerometers and gyroscopes to perform dead reckoning between laser beam sweeps [6].

The visual focus, referred to as "gaze", is captured using Tobii Pro Glasses 2. These eye-tracking glasses rely on retina geometry detection with infrared cameras to obtain a gaze vector for each eye. The resulting vectors from both eyes can be intercepted to obtain a spatial focus point or the individual vectors can be used separately [7].

The general architecture of the proposed system is presented in Figure 1. The 'model computer' (the computer system where the system's software is executed, it is referred to simply as "computer" in the next diagrams) receives information about the body gestures and gaze direction, as well as the aircraft states. Based on this information, it determines the aircraft control inputs.

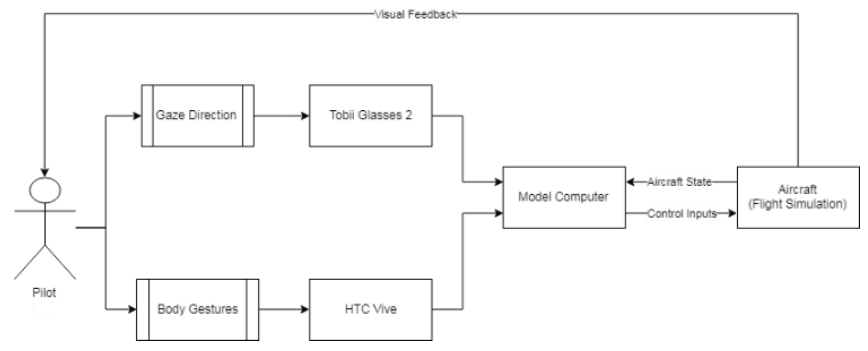

Figure 1: General diagram of the proposed system.

Next, we describe in detail each of the proposed aircraft interface.

\subsection{Eye Tracking System}

The Pro Glasses 2 measures the pilot gaze direction at a rate of $25 \mathrm{~Hz}$. It also provides the glasses acceleration and angular rates, measured by a Micro-Electro-Mechanical System (MEMS) Inertial Measurement Unit (IMU).

These variables are sent, via wi-fi, to the Tobii Software Development Kit (SDK). It is then packaged in an American Standard Code for information Interchange (ASCII)-coded string and sent using the Transfer Control Protocol (TCP) to the model computer. There, a control system implemented in Simulink interprets and processes the signals and generates a command output for the aircraft. The diagram in Figure 2 illustrates this.

The gaze vector is a two-component vector comprising the horizontal gaze and the vertical gaze component. Both components vary from 0 to 1 depending on the pilot's focusing direction. The $(0,0)$ coordinate is at the lower left corner of the pilot's field of view through the glasses, while the $(1,1)$ coordinate is at the top right corner.

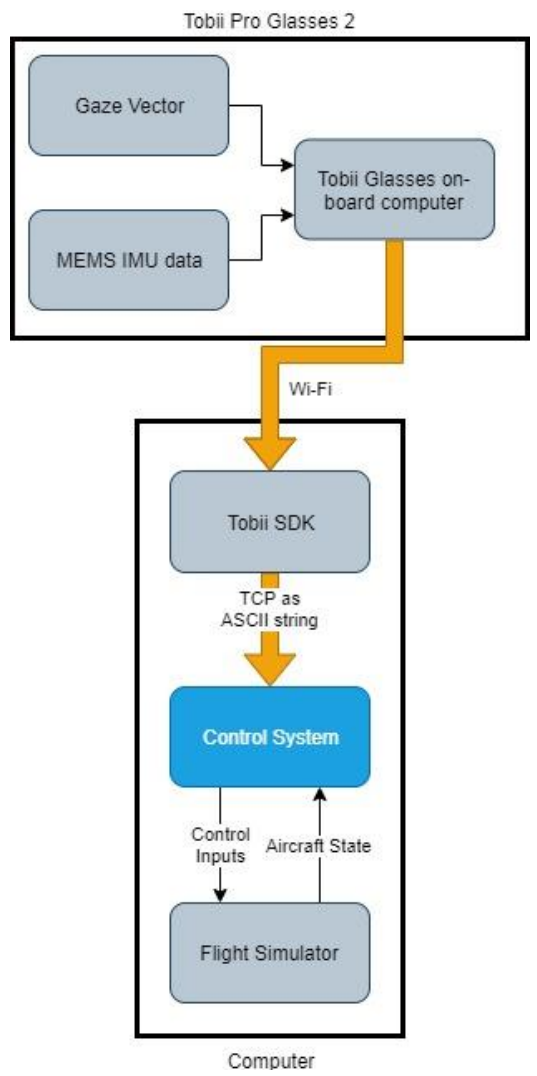

Figure 2: Gaze-based configuration architecture.

The gaze vector is fixed to the glasses reference system. Therefore, an additional reference is needed to convert this to a frame of reference aligned with the horizon. For this purpose, the IMU data is used to sense the orientation of the glasses with respect of the Earth's gravity. Combining the IMU data with the gaze vector provides us a gaze vector that is relative to the horizontal plane of the Earth instead of being relative to the glasses, irrespective of their inclination or orientation. Figure 3 illustrates the signal path in the control system, which is the blue block in Figure 2.

The current configuration allows the pilot to control the aircraft by looking into the desired trajectory. While focusing on the desire trajectory, he/she should press and release the 'command button'. This button assures that the gaze information is used to control the aircraft only when the pilot intends to do so.

When the pilot releases the command button, the system determines the gaze direction. It then processes it and defines the output for the aircraft' flight control surfaces, in order to guide the aircraft into the desired trajectory.

Before the first utilization of the system the pilot must define a "nose" vector for the aircraft. This is done by looking straight ahead to the display where the simulation will be shown and pressing a calibration button. The provided gaze vector is considered as a reference.

The above described operation mode is referred to as the "gaze-guided configuration" in the rest of the paper. 


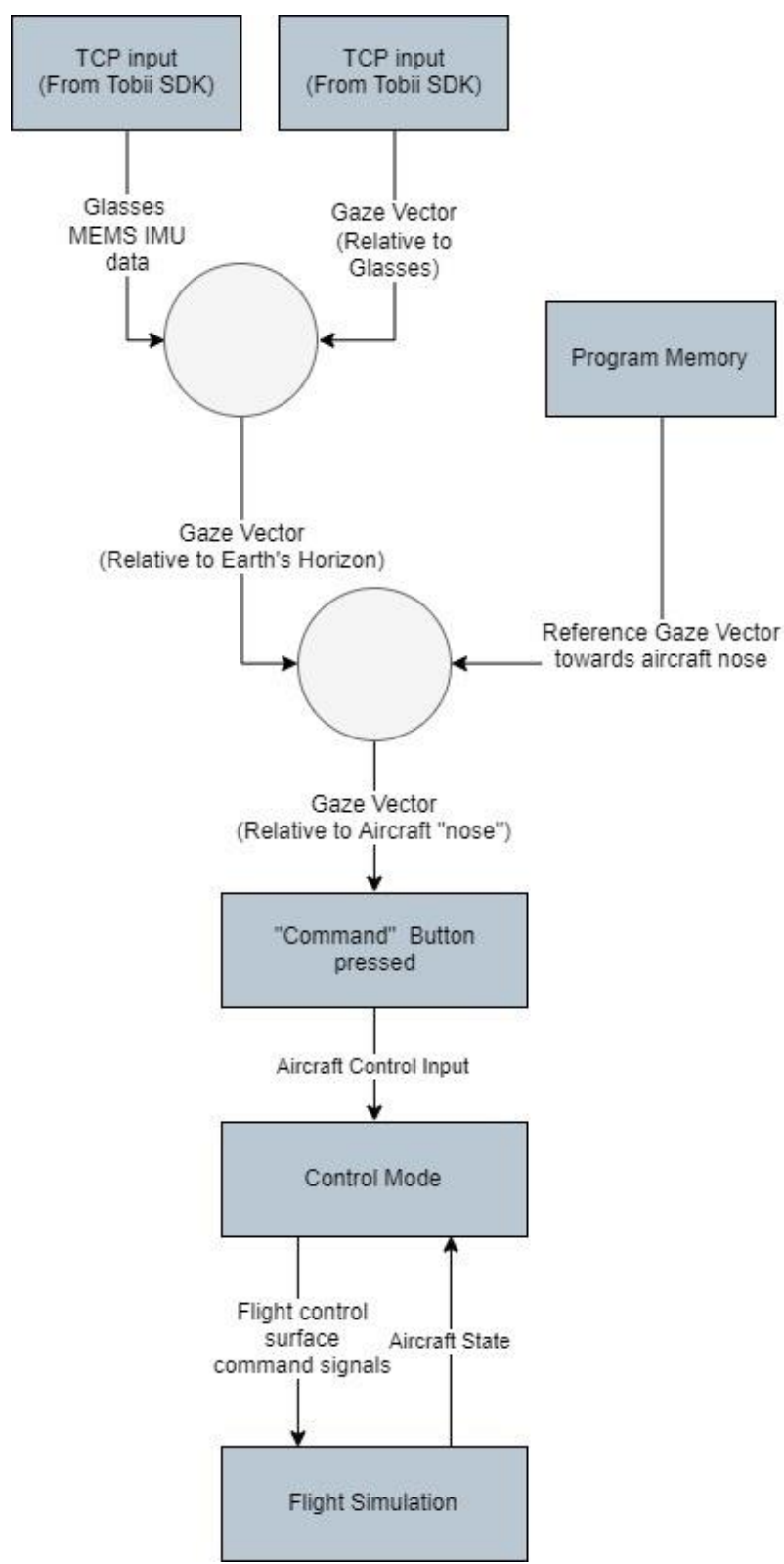

Figure 3: Gaze-guided configuration Control System.

\subsection{Gesture Tracking System}

The Vive is used at a $50 \mathrm{~Hz}$ tracking rate. Information from the lighthouse tracking stations is used to locate a Vive Controller in a reference frame. The horizontal axes of this frame are North-South and East-West. The vertical axis is in the Up-Down direction.

In order to obtain useful signals this reference is transformed to an arbitrary reference set by the pilot at the beginning of each usage of the system. This allows the roll input to be calculated using the longitudinal axis correspondent to the aircraft's own longitudinal axis instead of the North-South axis.

In the current setup, the controller is attached to the dorsal part of the pilot's hand eliminating the need to "hold" the controller. The Vive system then acquires tracking of this controller and feeds a 7-element vector to the SDK. This vector contains the three linear coordinates about the original
North-South East-West reference and the four quaternion rotation elements for the controller's current tracked state.

The SDK uses TCP to transmit this vector as a binary string to the Control System in Simulink, which performs the necessary transformations and calculates the input for the aircraft flight controls. This is illustrated in Figure 4.

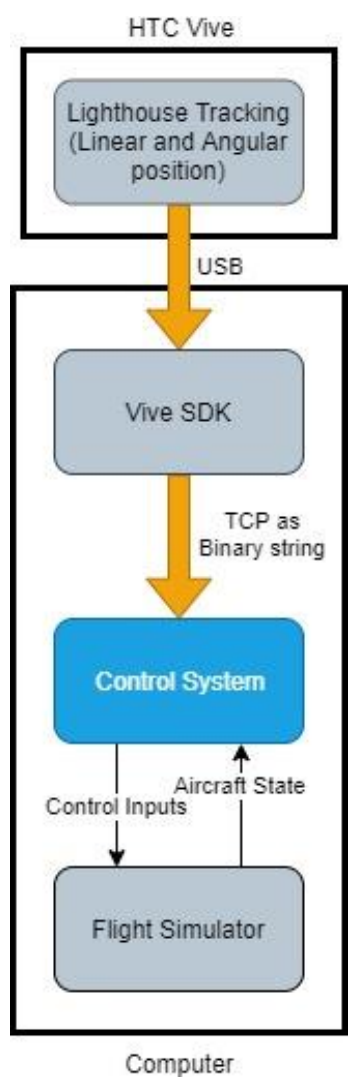

Figure 4: Hand-guided configuration architecture.

In the current configuration, the pilot "imitates" the aircraft using the hand, as a flight instructor would imitate aircraft rolls and climbs to illustrate maneuvers to students.

The system considers that the aircraft longitudinal axis extends along the open hand from the palm towards the middle finger. The nose of the aircraft is on the tip of this finger, while the left wing would extend towards the open thumb.

The system takes a roll of the hand as a roll input and a rise or lowering of the hand as a pitch input. This configuration will be referred to as the "hand-guided configuration" for the rest of the paper.

Like the gaze-guided configuration, the hand-guided configuration requires the pressing of a "command" button. The system reads the input only when the button is pressed and released. Figure 5 illustrates the signal path in the control system, which is the blue block in Figure 4. 


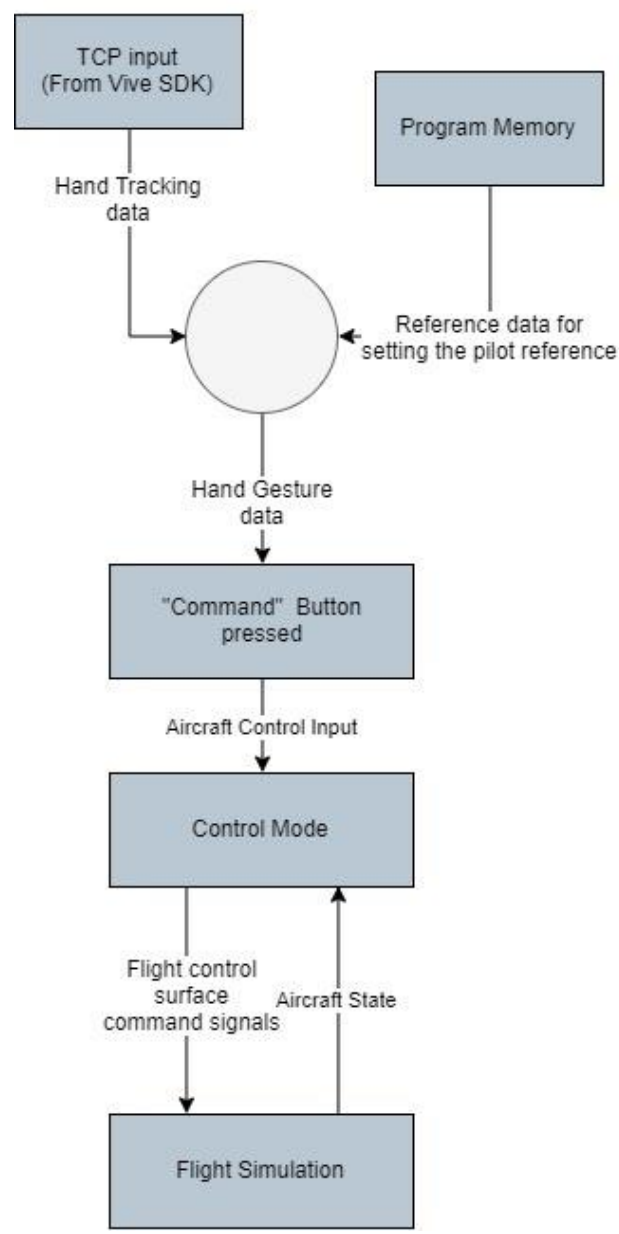

Figure 5: Hand-guided configuration control system

\section{Flight Control Modes}

A series of input signal interpretation modes are implemented in the control system in order to produce meaningful flight control signals based on body gestures and gaze direction. This set of control modes are only for initial exploratory purposes and might not be the most efficient or the most comfortable control modes possible for the system. All the input signals that these modes receive vary from -1 to +1 , where zero is a neutral input be it in roll or pitch.

\section{Flight Path Angle Mode}

This mode takes the processed input from either gaze or body tracking systems and makes this proportional to the aircraft's Flight Path Angle (FPA) or Flight Path Trajectory.

The input is passed through a gain that converts it to a target FPA for a controller to track it by commanding elevator deflection.

\section{Flight Path Angle Change Mode}

Similar to the previous mode, but it instead makes the new FPA to be a function of current FPA at the time of the command being issued. It adds an angle proportional to that of the received input signal.

\section{Roll Angle Mode}

In this case, the input signal is passed through a gain that converts it to a specific roll angle to either side. A controller will then track this reference generating aileron deflection signals.

\section{Heading Change Mode}

This mode allows for the pilot to look or point with the hand in a direction to either side of the aircraft's nose and set a new heading.

The system takes the current heading at the time of the command being issued and computes the angle between the nose of the aircraft and the direction that the pilot is gazing or pointing at. It then stablishes the new heading as the current heading plus this difference.

Finally, a controller tracks the set heading by generating outputs for the ailerons up to a specific maximum roll angle.

\section{Experimentation}

A series of simple simulated flights were performed to acquire an initial experience with the system and provide a better understanding of it. The information collected will be used to improve the system from its current state.

As a way of simplifying inputs, only either roll or pitch is controlled at a time during each flight. This allows the pilot to focus on a single type of input signal. It also results in a set of data that is more easily interpreted and analyzed for each configuration and control mode.

\subsection{Experiments Set-up}

Even though the system intends to explore the feasibility of a gesture-based system for piloting a manned aircraft, the initial experiment uses the Flight Dynamics Model (FDM) of a light cargo Unmanned Aerial Vehicle (UAV) for research timeefficiency purposes. Figure 6 provides a rendering of this aircraft.

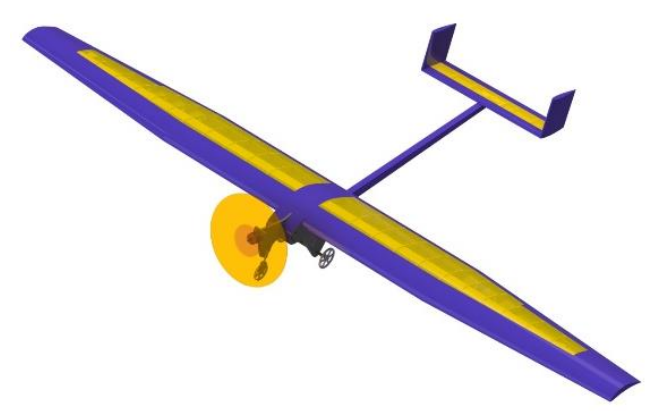

Figure 6: Rendering of the Aircraft model used during the experiment.

The Aircraft contained in this flight simulator had its FDM verified using wind tunnel and flight test data and is considered an appropriate representation of the real equivalent, it has Electric propulsion producing 40 Newtons of static trust, a mass of 15 Kilograms and a Wingspan of 3.2 
meters. The fact that it is modeled in Simulink allows for a quick integration into the rest of the system.

Being a simulation, it is possible to place the pilot's perspective to be "inside" of the aircraft, therefore providing for the same type of perceptions as a manned aircraft. Further work on the next round of experiments is expected to be carried out using FDMs of manned transport or acrobatic aircraft.

The output spatial position and Euler angles representing the aircraft attitude in space is calculated in Simulink and sent to the FlightGear flight simulator. Flight Gear then provides a visual feedback of the aircraft's flight trajectory to the pilot.

The simulated flight tests presented in this paper take place near the Sao Jose dos Campos Airport (SBSJ) in Brazil.

\subsection{Scenario 1: Aligned Approach for Landing}

The aircraft begins the flight aligned with a runway at a distance of $2 \mathrm{~km}$ from the threshold. It has an altitude that places it near the typical 3-degrees glideslope for landing.

The pilot is tasked with controlling only the longitudinal axis of the aircraft in order to intercept and maintain the glideslope for landing as close as possible to the runway's aiming marks, as illustrated in Figure 7. The flight ends as soon as the aircraft contacts with the runway, recording the position where touchdown occurred for later analysis.

The roll and yaw in this flight are fixed to have no variation during the experiment while the airspeed is kept constant by an auto throttle system.

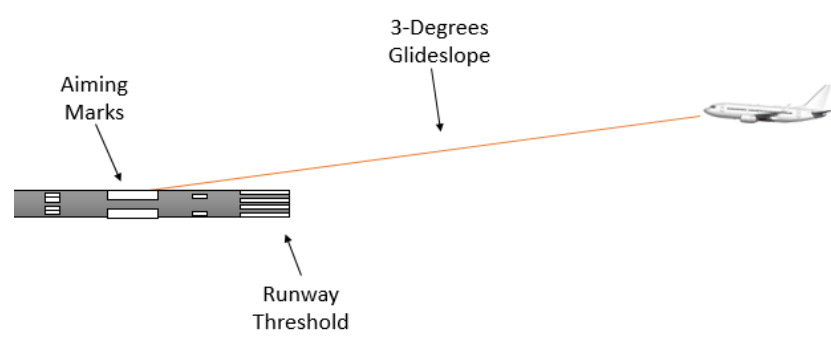

Figure 7: Aligned approach for landing.

The purpose of this flight is to evaluate the precision control of the longitudinal axis of the aircraft using both gaze-guided and hand-guided configurations.

The distance from the touchdown point and the aiming marks of the runway as well as the trajectory of the aircraft relative to the 3-degrees glideslope are used as indicators of performance. The closer that the touchdown occurs to the aiming point and the smaller the deviation from the glideslope are considered to indicate a higher performance.

\subsection{Scenario 2: Non-Aligned Approach for Landing}

The aircraft begins the flight 2.8 kilometers from the Runway threshold. It is at an altitude that allows it to align and then land on that runway on a 3-degrees glideslope. In this case the aircraft is heading towards the threshold at the beginning of the flight but is not aligned with the runway heading.
The pilot is tasked with controlling only the roll axis of the aircraft in order to intercept and maintain the runway heading while the vertical speed is adjusted to allow the aircraft to land on the runway.

If the pilot handles lateral guidance correctly, the flight ends when the aircraft touches down on the runway. At this point the position where touchdown occurred as well as the heading of the aircraft at that moment is recorded for later analysis.

Figure 8 illustrates the flight of this scenario, this flight was adapted from reference [8] where a similar scenario is presented for evaluation of a brain-controlled interface.

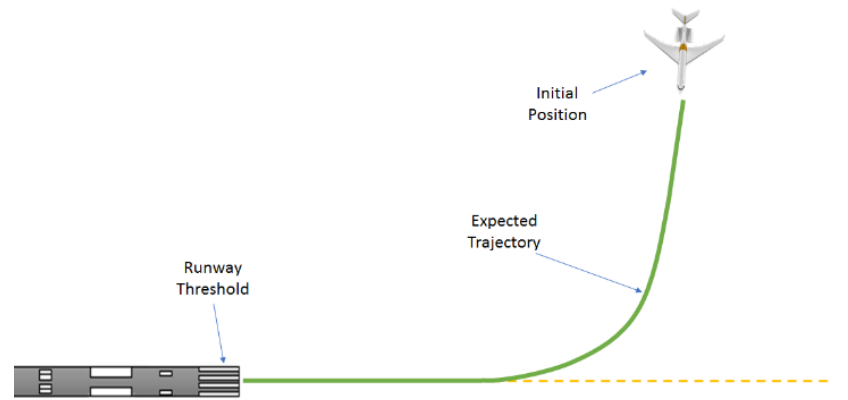

Figure 8: Reference flight for non-aligned approach.

The purpose of this flight is to evaluate the precision control of the aircraft in the roll axis using the proposed interfaces.

Touchdown position relative to aiming point and the aircraft's heading at the time of the touchdown are used as metrics for performance in this flight. Smaller deviations from expected touchdown point and runway heading at touchdown indicate better performance.

\section{Results}

The two scenarios described in section 4 where performed with the aircraft model of section 4.1.

The flights of Scenario 1 where performed with the pilot controlling the longitudinal axis only and is referred to as "Vertical Guidance". The flights of Scenario 2 were performed with the pilot controlling only the laterodirectional flight and is referred to as "Horizontal Guidance" scenario. In each case the pilot used either the gaze or hand gesture guidance configurations.

Each flight scenario with each interface configuration was repeated 5 times by a single pilot, for a total of 20 flights performed with the intention of gaining insight of the system's operation.

\subsection{Vertical Guidance}

Firstly, the gaze-guided configuration was used with the FPA change mode. The results are presented in Figure 9, where the dashed red line represents the ideal glideslope.

It shows that the gaze-based configuration required constant corrections to be performed by the pilot. In some flights, the flight path is abruptly inverted. this problem could be related to the accuracy of the system calibration. Approaches to improve it are currently under investigation. 


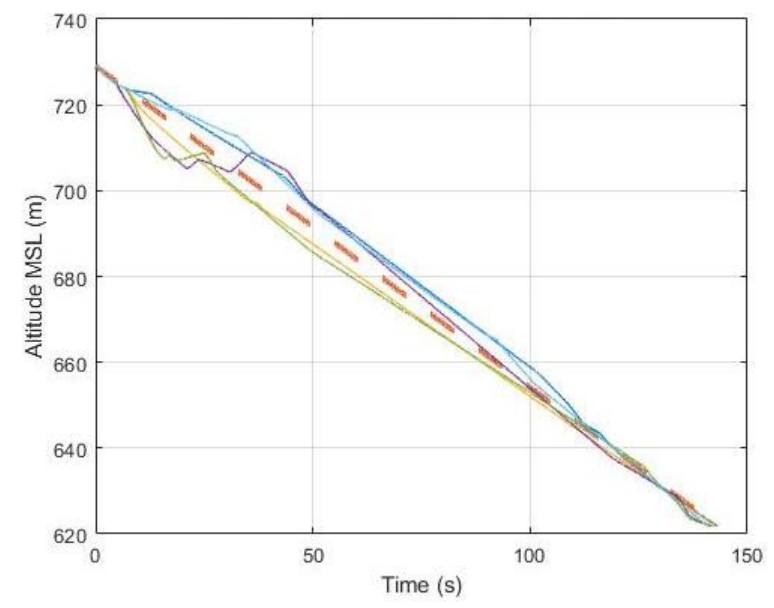

Figure 9: Altitude for five gaze-guided landings.

Next, the hand-guided configuration was used with the FPA proportional mode. Results are presented in Figure 10, where the dashed red line represents the ideal glideslope. The handbased configuration allowed for a more precise control of the landing when compared to gaze-guided landing as can be seen in. It also presented a lower number of inversions of the flight path angle when compared to those on the gaze-guided configuration.

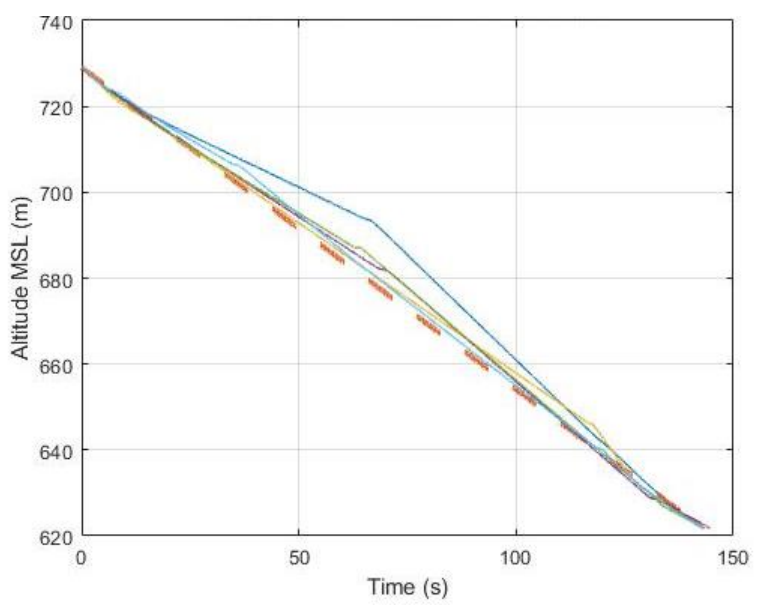

Figure 10: Altitude for five hand-guided landings.

Figure 11 shows an example of the FPA set by the pilot using gaze-guided input. Figure 12 shows the partially processed input of a hand-guided flight.

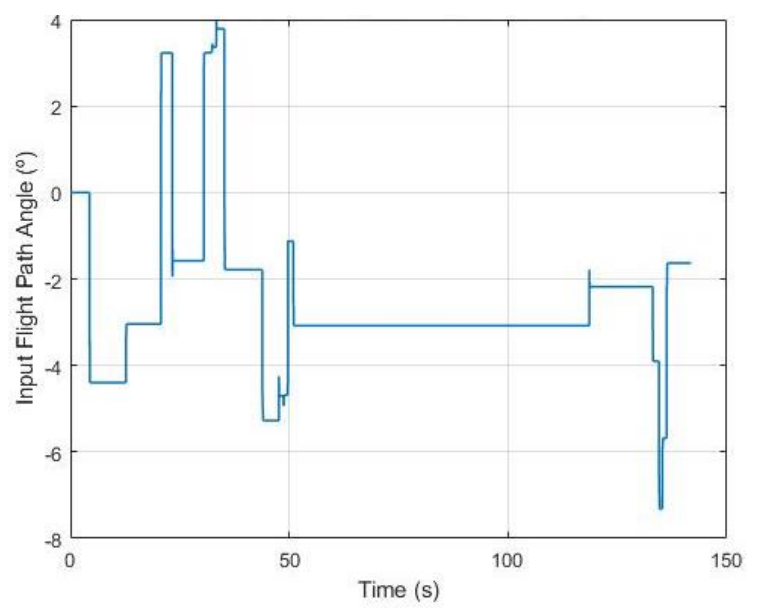

Figure 11: Gaze-guided FPA signal during one of the test flights.

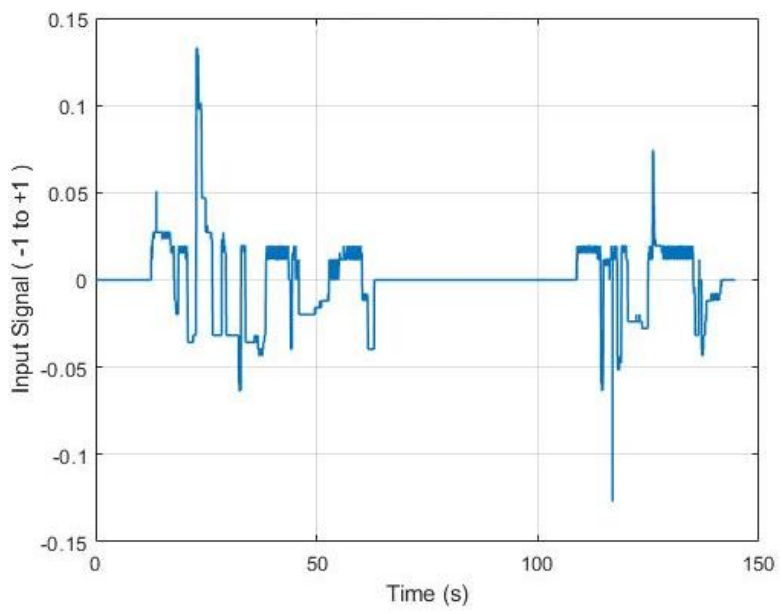

Figure 12: Hand-guided input signal before being converted to FPA during one of the test flights.

\subsection{Horizontal guidance}

The horizontal guidance flights had the limitation that the field of view on the computer's screen did not allow for visualization of the runway when the aircraft was perpendicular to it. Therefore, in both gaze-guided and handguided configurations, the pilot had to make turns into the runway to verify its position relative to it, and then return to its previous trajectory.

For this flight, the gaze-guided configuration used the heading change mode while the hand-guided configuration used the roll-proportional mode.

Both the gaze-guided and hand-guided configurations showed enough accuracy on the guidance of the aircraft into the runway heading to perform a landing on the aiming points of the runway. Figure 13 shows the trajectory for all five gaze-guided landings while Figure 14 presents the results for the hand-guided landings. 


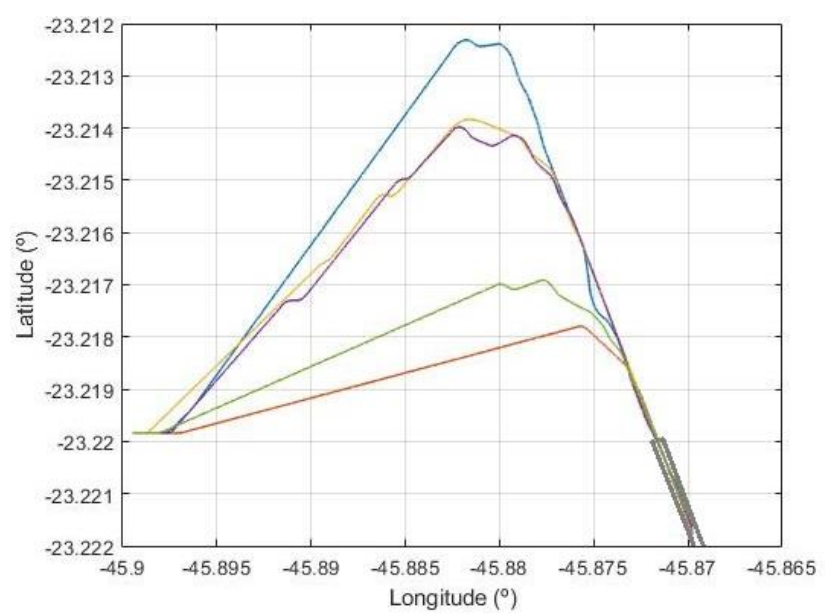

Figure 13: Horizontal trajectory of the aircraft during the gaze-guided landings.

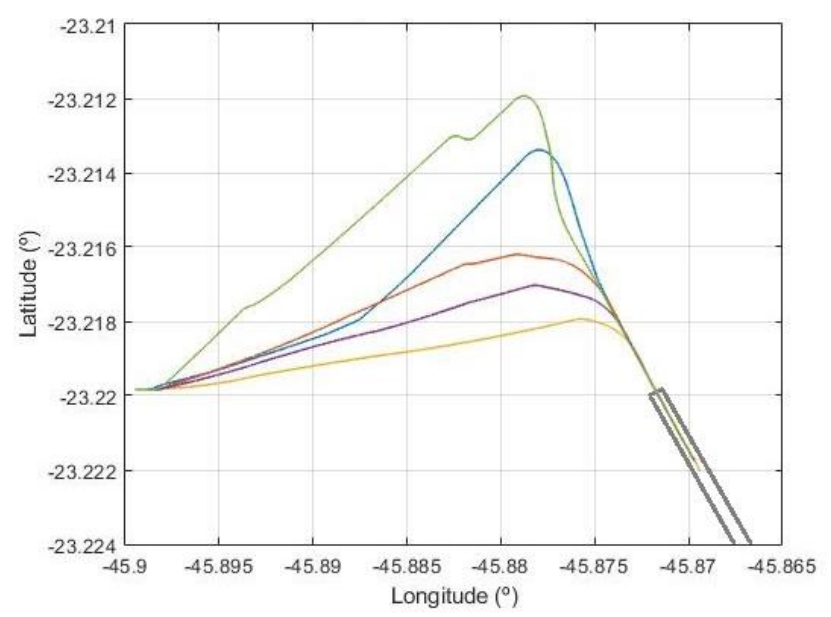

Figure 14: Horizontal trajectory of the aircraft during the hand-guided landings.

Signals recorded during these flights are presented in Figure 15 for the gaze-guided heading and in Figure 16 for the handguided configuration.

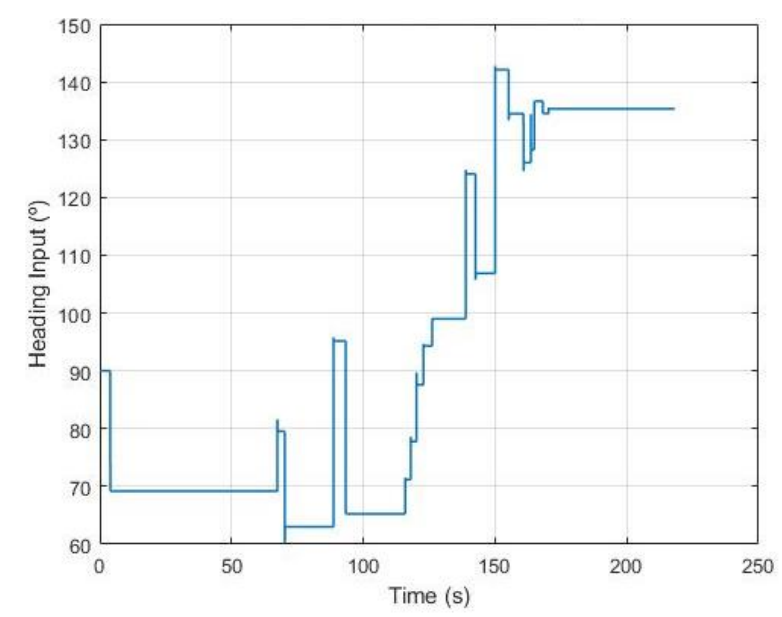

Figure 15: Gaze-guided heading input for landing during one of the test flights.

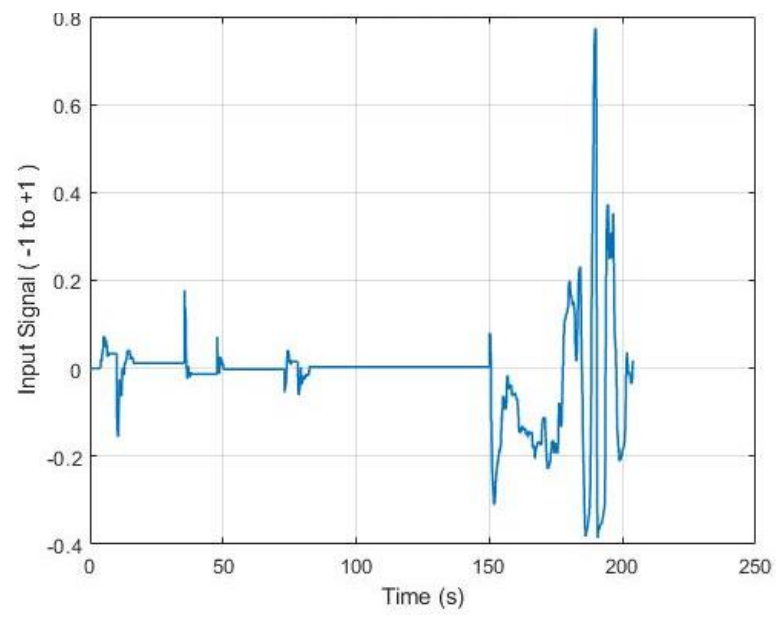

Figure 16: Hand-guided inputs before being converted to Roll inputs during one of the test flights.

\subsection{Data Analysis}

Figure 17 presents the error in the runway touchdown position when compared with the expected position, for the four cases analyzed in this paper. Although some variation can be observed in Figure 17, it is not possible affirm that one of them is significantly better than the other.

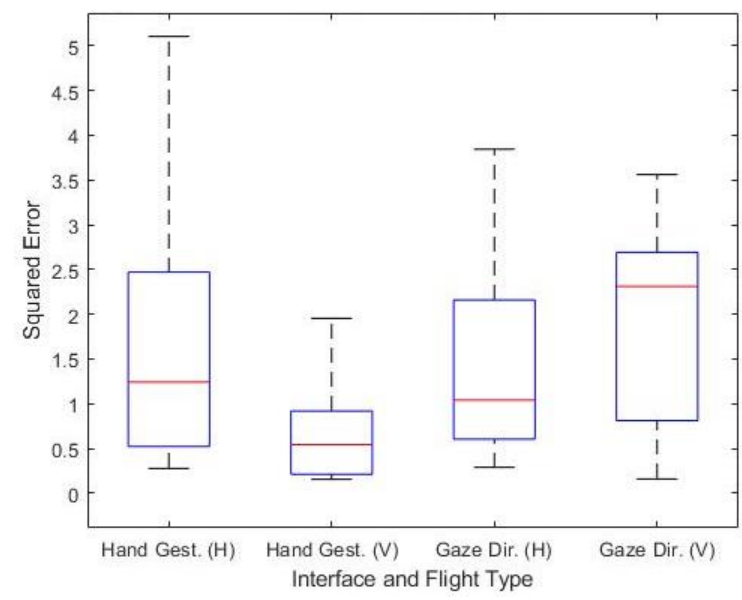

Figure 17: Touchdown position error for each case.

On the other hand, Figure 18 shows that in the current experiment the gaze-guided configuration presents a greater deviation from expected flight trajectories during approach (deviation from the expected glideslope) compared to those of the hand-guided configuration. 


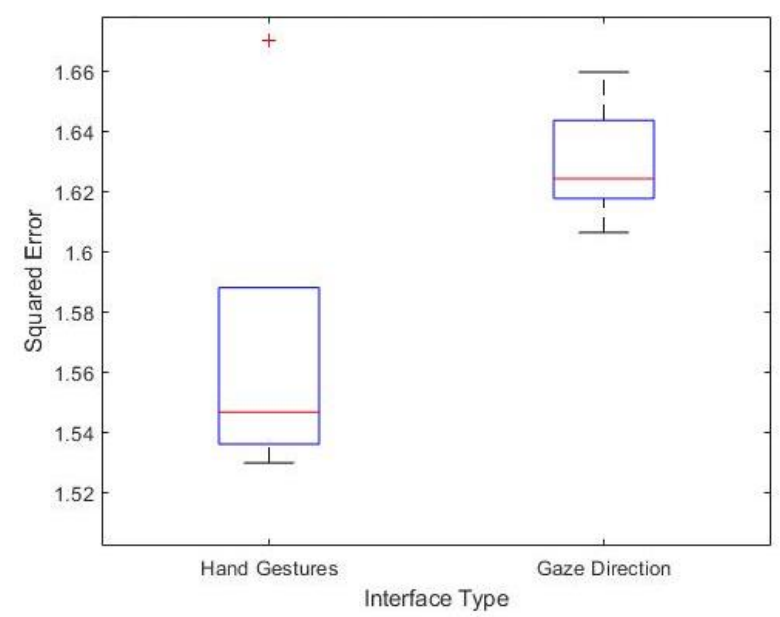

Figure 18: Cumulative glideslope deviation for the vertical guidance flight tests.

The final heading at touchdown also shows better performance with the hand-guided configuration as can be seen in Figure 19. This can also be attributed to the early development stage of the gaze-based configuration.

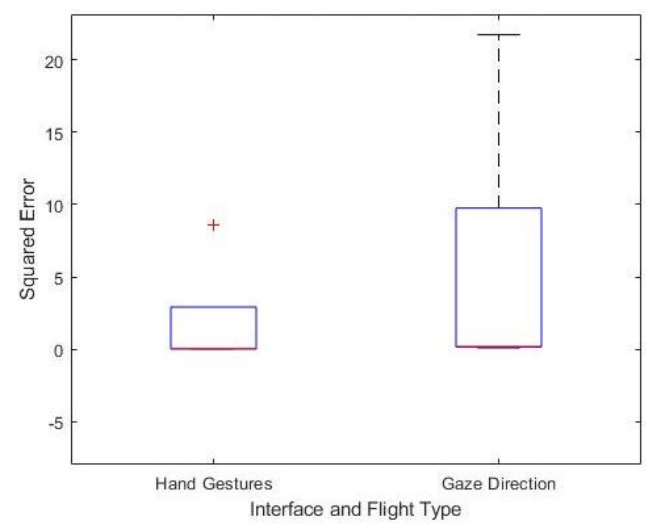

Figure 19: Aircraft heading error at touchdown for horizontal guidance test flights.

It is also clear from Figure 20 that the method of using both configurations is different. The gaze-guided configuration calls for a quick "stare" into the desired trajectory and then to issue a single pressing of the "command" button to allow the aircraft to follow that trajectory, making quick adjustments as required.

On the other hand, the hand-guided configuration allows the pilot to press the "command" button for longer periods, having direct and precise control of the trajectory for longer periods of time. In fact, it requires this method of input to achieve the landing.

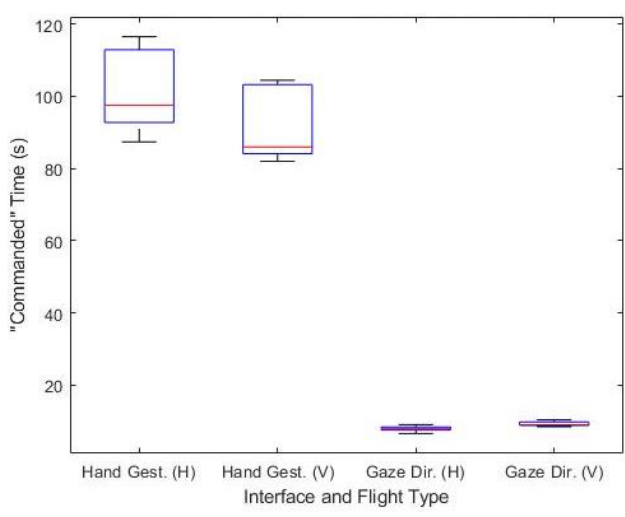

Figure 20: Cumulative "command" button time pressed for all the test scenarios and configurations.

It can also be seen that the necessity to use a "command" signal was confirmed. Figure 21 shows the uninhibited gaze signal as received by the system. Figure 11 shows the obtained command from that signal as the "command" button was pressed and released. Not having such an interruption makes the aircraft uncontrollable as the pilot would have to keep constant focus on the desired flight trajectory, even with the hand-guided configuration.

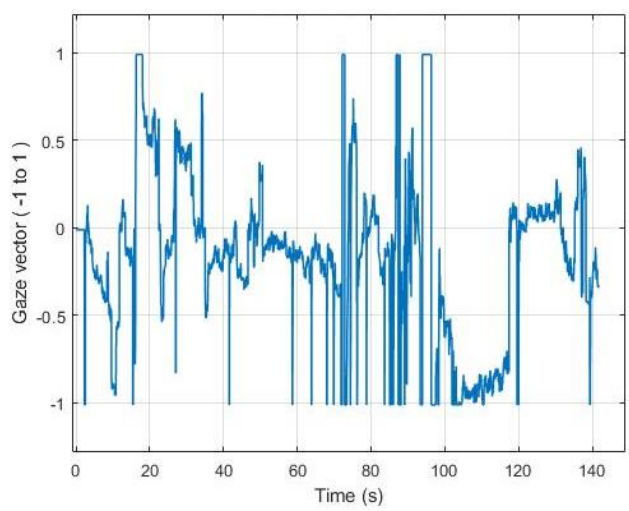

Figure 21: Example of a vertical gaze signal before being isolated by the "command" button during a whole flight.

\section{Conclusions and Future Work}

In this paper, the feasibility of gaze-guided and hand-guided control of an aircraft was demonstrated. Data collected in a first set of experiments will be used for further development of flight control modes, aiming at improving system performance.

Operational experience gained with this experiment allows for the planning of elaborate experiments using different pilots that are not yet accustomed to the system. New experiments would allow to properly measure the impact of the proposed interfaces on simulated flight.

Follow-up experiments will be conducted in a highly realistic flight simulator that includes wide field of view and a complete cockpit environment. It will also use a transportclass manned aircraft model and elaborate flight scenarios that would allow to compare the proposed interfaces with "classical" interfaces such as yokes and sidesticks in the same environment. 


\section{References}

[1 J. Roskam, Airplane Design, Lawrence: DAR

] Corporation, 1985.

[2 NASA Armstrong, "X-29 Advanced Technology

] Demonstrator Aircraft," [Online]. Available: http://www.nasa.gov/centers/armstrong/news/FactSheet s/FS-008-DFRC.html.

[3 Airbus, "Vahana, the Self-Piloted, eVTOL aircraft from

] $\mathrm{A}^{3}$ by Airbus, Successfully Completes First Full-Scale Test Flight," 02 February 2018. [Online]. Available: https://www.airbus.com/newsroom/pressreleases/en/2018/02/vahana--the-self-piloted--evtolaircraft-from-a--by-airbus--succ.html. [Använd 1208 2019].

[4 Embraer, "EmbraerX unveils first eVTOL concept," 5

] August 2018. [Online]. Available: https://embraer.com/global/en/news/?slug=906344embraerx-unveils-first-evtol-concept. [Använd 1208 2019].

[5 The Boeing Company, "Boeing Autonomous Passenger

] Air Vehicle Completes First Flight," 23 January 2019. [Online]. Available: boeing.com/features/2019/01/pavfirst-flight-01-19.page. [Använd 12 August 2019].

[6 D. C. Niehorster, L. Li och L. Markus, "The Accuracy ] and Precision of Position and Orientation Tracking in the HTC Vive Virtual Reality System for Scientific Research," i-Perception, nr May-June, pp. 1-23, 2017.

[7 Tobii, "Pro Glasses 2 Product Description," Tobii AB, ] 2018.

[8 T. Fricke, "FIRST PILOT-IN-THE-LOOP ] SIMULATOR EXPERIMENTS ON BRAIN CONTROL OF HORIZONTAL AIRCRAFT MOTION," Deutscher Luft- und Raumfahrtkongress, 2014. 